\title{
ISOMORPHISMS OF THE ENDOMORPHISM RINGS OF A CLASS OF TORSION-FREE MODULES
}

\author{
KENNETH G. WOLFSON ${ }^{1}$
}

Introduction. In [7] and [8] we have determined the isomorphisms of the endomorphism rings of two special classes of torsion-free modules. In [7] the modules were free, over principal left ideal domains, and the isomorphism was always induced by a semi-linear transformation of the modules. In [8] the ring of scalars was a complete discrete valuation ring, but no further restriction was placed on the modules. Except for the special case when both modules are divisible, the same result holds as in [7]. In each case a key point in the argument is that a certain indecomposable direct summand can be shown to be cyclic. Even for ordinary torsion-free abelian groups, the abundance of the indecomposable ones makes it impossible to prove that isomorphism of the endomorphism rings implies isomorphism of the underlying groups. We show first that for any cardinal number $c$, there exist homogeneous completely decomposable groups $G$ and $H$ of rank $c$ which are not isomorphic, but have isomorphic rings of endomorphisms. This makes it clear that strong restrictions must be placed on the class of groups considered if it is hoped that the ring isomorphism is to be induced by a group isomorphism. In particular, homogeneity coupled with separability or complete decomposability is not sufficient.

We are, however, able to generalize the results of [7] to a class of what we call locally free modules $A$ over principal left ideal domains $F$. A locally free abelian group ( $F$ is the ring of integers) is just a homogeneous separable group of null type [6, p. 208]. This latter class of groups includes the complete direct sum of infinite cyclic groups, as well as all its subgroups [4, p. 176]. For example, the set of all bounded sequences of integers is such a group.

We also determine (Theorem B) which semi-linear transformations induce the same ring isomorphisms. The results are similar to the vector space case considered in [2], and apply to the endomorphism rings of locally free modules as well as the torsion-free modules of [8].

1. An example and some preliminaries. Let $G_{1}$ be the additive group of integers, and let $H_{1}$ be the additive group of those rational

Received by the editors February 23, 1962.

1 This paper was written while the author held grant NSF-G 19053 from the National Science Foundation. 
numbers which when written in lowest terms have square free denominators. Now $G_{1}$ has the type $(0,0,0, \cdots)$ while $H_{1}$ has type $(1,1,1, \cdots)$. Since these are distinct types, $G_{1}$ and $H_{1}$ are not isomorphic [4, Theorem 42.2, p. 149]. The endomorphism ring of $G_{1}$ is isomorphic to the ring of integers [4, Example 1, p. 211]. The endomorphisms of $H_{1}$ consist of multiplications by rational numbers $r$ [4, Examples 3, 4, p. 211]. But only those rationals $r$ are permissible for which $r H_{1} \subseteq H_{1}$. Hence $r$ when written in lowest terms can contain no primes in its denominator. Thus the endomorphism ring of $H_{1}$ is also isomorphic to the ring of integers.

Let $c$ be any finite or infinite cardinal, and let $G$ and $H$ be the direct sums of $c$ copies of $G_{1}$ and $H_{1}$ respectively. Then $G$ and $H$ are nonisomorphic homogeneous completely decomposable groups of rank $c[4$, Theorem 46.1]. The endomorphism rings of $G$ and $H$ are, however, isomorphic [4, Theorem 55.1].

Hence it does not follow from the isomorphism of the endomorphism rings that the underlying groups are isomorphic, even if one assumes that the groups in question are homogeneous, and also separable or even completely decomposable (cf. [4, Problem 43, p. 232]). (In the case of completely decomposable groups the arguments of [7, Lemma 2.2] will, however, allow the conclusion that the ranks are equal. Hence if the groups are assumed homogeneously completely decomposable and of the same type, then the group isomorphism follows from the ring isomorphism.)

Let $(F, A)$ be a torsion-free module $A$ over a principal left ideal domain $F$. We shall call $(F, A)$ locally free if each finite subset of $A$ is contained in a free direct summand. It is clear that we may assume that this direct summand has finite rank.

We shall follow the definitions and notations of [7]. In particular, $E(F, A)$ is the endomorphism ring of $(F, A)$ and $E_{0}(F, A)$ consists of the endomorphisms of finite rank. Our result is as follows.

Theorem A. Let $(F, A)$ and $(G, B)$ be locally free modules over principal left ideal domains. If $\sigma$ is an isomorphism of $E(F, A)$ upon $E(G, B)$ then there exists a one-to-one semi-linear transformation $\omega$ of $(F, A)$ upon $(G, B)$ such that $\eta^{\sigma}=\omega^{-1} \eta \omega$ for each $\eta \in E(F, A)$.

We shall prove a few preliminary results and then show how to modify the arguments of [7] to get the desired result. If the rings of scalars were assumed both left and right principal ideal domains, some of the arguments would be simpler since over such rings, finitely generated, torsion-free modules are all free [5, Theorem 18, p. 44]. 
Lemma 1.1. Let $(F, A)$ be locally free, and $S$, a submodule of finite rank. Then $S$ is contained in a free direct summand of finite rank and is thus itself free of finite rank.

Proof. Let $x_{1}, x_{2}, \cdots, x_{n}$ be a maximal linearly independent set in $S$. Then $\sum_{i=1}^{n} F x_{i} \subseteq Q$, a free direct summand of finite rank. If $x \in S$, then $r x \in \sum_{i=1}^{n} F x_{i} \subseteq Q$ for some $r \neq 0$ in $F$. But $Q$ being a direct summand, is pure, so $x \in Q$. Hence $S \subseteq Q$, and $S$ is free as a submodule of a free module [3, Theorem 5.3, p. 13].

It now follows easily that $E_{0}(F, A)$ is a two-sided ideal of $E(F, A)$.

Lemma 1.2. If $(F, A)$ is locally free, and $S$ is a direct summand of $A$, then $(F, S)$ is locally free.

Proof. Let $A=S \oplus Q$ with $a_{1}, a_{2}, \cdots, a_{n} \in S$. Then by hypothesis the $a_{i} \in W$, with $A=W \oplus Z$ and $W$ free of finite rank. Now $W / S \cap W$ $\cong(S+W) / S$. Since $W$ is finitely generated, the same is true of $(S+W) / S$. But $S$ is a direct summand of $S+W$, since it is a direct summand of $A$. Thus $(S+W) / S$ is isomorphic to a submodule of $S+W$, hence to a submodule $T$ of $A$. But $T$ is finitely generated, hence of finite rank, so free by Lemma 1.1. Therefore $W / S \cap W$ is free.

So by $[4$, Theorem 9.2, p. 38] $S \cap W$ is a direct summand of $W$. Hence $S \cap W$ is a direct summand of $A$, since $W$ is a direct summand of $A$. This gives finally that $S \cap W$ is a direct summand of $S$. This completes the proof since the $a_{i} \in S$, and $S \cap W$ is free as a submodule of the free module $W$.

REMARK. It follows from Lemma 1.2 that an indecomposable direct summand of a locally free module must be cyclic. If the rings of scalars $F$ and $G$ are assumed commutative, a proof of the theorem could now be given by the method of [8].

2. The isomorphism theorem. In this section we shall number our Lemmas to correspond to the similar ones proved in [7].

Lemma 2.2. Suppose $E(F, A) \cong E(G, B)$ with $(F, A)$ free of finite rank $n$, and $(G, B)$ locally free. Then $(G, B)$ is free of rank $n$.

Proof. If $B$ has finite rank, it follows from Lemma 1.1, that it is free, so the proof would follow from Lemma 2.2 of [7]. Suppose it is possible that $B$ has infinite rank. Let $x_{1}, x_{2}, \cdots, x_{n+1}$ be linearly independent elements of $B$ and imbed them in a free direct summand $P$ of rank $m \geqq n+1$. Let $y_{1}, y_{2}, \cdots, y_{m}$ be a basis of $P$ and let $e_{i}, i=1,2, \cdots, m$ be the projection on $G y_{i}$ which annihilates the other basis elements and the complementary summand. If $f_{i} \rightarrow e_{i}$ under the isomorphism, then 


$$
r(A) \geqq r\left(\sum_{i=1}^{m} A f_{i}\right) \geqq m,
$$

a contradiction.

Lemma 2.3. Let $\alpha$ be an isomorphism of $E(F, A)$ on $E(G, B)$, then $\alpha$ induces an isomorphism of $E_{0}(F, A)$ on $E_{0}(G, B)$.

Proof. We imitate the corresponding proof in [7]. If $A \sigma=S$, $S \subseteq Q$, where $Q=A e$ is a free direct summand of finite rank $k$, by Lemma 1.1. Then we have $E(F, A e) \cong E(G, B f)$, where $B f$ is locally free by Lemma 1.2. The result now follows from Lemma 2.2.

Lemma 2.4. Let $\phi$ be an automorphism of $E(F, A)$ which leaves the elements of $E_{0}(F, A)$ elementwise fixed. Then $\phi$ is the identity.

Proof. Let $\sigma \in E(F, A)$. If $a \in A$, imbed it in a free direct summand of finite rank, and let $e_{a}$ be the projection on it, so that $e_{a} \in E_{0}$. Then

$$
e_{a} \sigma^{\phi}=e_{a}^{\phi} \sigma^{\phi}=\left(e_{a} \sigma\right)^{\phi}=e_{a} \sigma .
$$

Hence $a \sigma^{\phi}=a e_{a} \sigma^{\phi}=a e_{a} \sigma=a \sigma$, so that $\sigma=\sigma^{\phi}$.

Before the proof of the next result we recall briefly some of the relevant notations of [7].

The set of all $F$-homomorphisms of $A$ into $F$ forms a right $F$ module (the adjoint module) denoted by $\left(A^{*}, F\right)$. If $x \in A$, and $y \in A^{*}$, the effect of the homomorphism $y$ on the element $x$ is denoted $(x, y)$. The pair $\left(A, A^{*}\right)$ is dual if $(x, y)=0$, for all $y \in A^{*}$, implies $x=0$. If $S$ is a subset of $A$, its annihilator in $A^{*}$ is the submodule $S^{\prime}$ of elements $f \in A^{*}$, for which $(S, f)=0$. If $T \subseteq A^{*}$, we similarly define $T^{\prime}$, the annihilator of $T$ in $A$. A submodule $Q$ of $A$ or $A^{*}$ is called closed if $Q^{\prime \prime}=\left(Q^{\prime}\right)^{\prime}=Q$. For $S$ a subset of $A, R(S)$ is the totality of $\rho$ in $E_{0}$ for which $S \rho=0$, and $L(S)$ is the set of $\alpha$ in $E_{0}$ for which $A \alpha \subseteq S$. If $P$ is a subset of $E_{0}(F, A)$, then $N(P)$ is the totality of $x$ in $A$ for which $x P=0$, and $A P$ is the set of elements $a p$, for $a \in A, p \in P$.

Lemma 3.1. (a) $A L(S)=S$ for each submodule $S$ of $A$.

(b) $N[R(S)]=S$ if and only if $S$ is a closed submodule of $A$.

Proof. (a) There exists a cyclic direct summand $F x, A=F x \oplus Q$. If $s \in S$, define $\sigma \in E_{0}$ by $x \sigma=s$ and $Q \sigma=0$. Then $\sigma \in L(S)$, and $s \in A L(S)$ giving $S \subseteq A L(S)$.

In particular we note that $A E_{0}=A L(A)=A$.

(b) We need only show that $\left(A, A^{*}\right)$ form a dual pair. If $a \in A$, $a \neq 0$, imbed $a$ in free direct summand with basis $b_{1}, b_{2}, \cdots, b_{n}$. Then $a=\sum_{i=1}^{n} r_{i} b_{i}$ with $r_{1} \neq 0, r_{i} \in F$. 
Define $f \in A^{*}$ by $\left(b_{1}, f\right)=1,\left(b_{i}, f\right)=0, i \neq 1$ with $f$ annihilating the complementary summand. Then $(a, f)=r_{1} \neq 0$ completing the proof.

The rest of the assertions of Lemma 3.1 of [7] as well as those of Lemmas 3.2, 3.3 and 3.4 follow as before.

Lemma 3.5. Suppose $r(A) \geqq 2$. If $\phi$ is an automorphism of $E_{0}(F, A)$ which leaves each left ideal invariant, $\phi$ is the identity.

Proof. Let $a \in A$ be arbitrary, and contained in a direct summand with basis $b_{1}, b_{2}, b_{3}, \cdots, b_{n}$. Let $b_{1}=b$ and $g$ be arbitrary in $A$. Let $\alpha(b, g) \in E_{0}$, map $b$ onto $g$, annihilate $b_{2}, \cdots, b_{n}$ as well as the complementary summand. Following the arguments of [7] we have $\alpha(b, g)=\alpha^{\phi}(b, g)$ if $b$ and $g$ are linearly independent.

Now suppose $b, g$ are dependent so that $s g \in F b$ for some $s \neq 0$ in $F$. But then $g \in F b$, since $F b$ being a direct summand is pure. We have $g=r b, r \neq 0$, in $F$ and may follow the arguments of $[1$, p. 199] to conclude $\alpha^{\phi}(b, r b)=\alpha(b, r b)$ so that $\alpha^{\phi}(b, g)=\alpha(b, g)$ for any $g \in A$. If $\delta \in E_{0}$, the last paragraph of the aforementioned proof shows $\delta$ and $\delta^{\phi}$ have same effect on $b=b_{1}$. It follows in the same way that $\delta$ and $\delta^{\phi}$ have the same effect on all the $b_{i}$; and hence on $a$. Since $a$ is arbitrary $\delta=\delta^{\phi}$, and $\phi=1$.

The proof of Theorem A may now be completed, by following the arguments of [7] without change.

Theorem B. Let $(F, A)$ and $(G, B)$ be torsion-free modules over principal left ideal domains, such that each possesses a cyclic direct summand, and $r(A) \geqq 2$. If $\sigma$ and $\tau$ are semi-linear transformations of $(F, A)$ upon $(G, B)$, then the following statements are equivalent:

(i) $\sigma$ and $\tau$ induce the same ring isomorphism of $E(F, A)$ upon $E(G, B)$,

(ii) $S^{\sigma}=S^{\tau}$ for each submodule $S$ of $A$,

(iii) There exists a unit $g$ in $G$ such that $x^{\sigma}=g x^{\tau}$ for each $x \in A$.

Proof. If we assume (i), Lemma 3.4 of [7] gives us $L\left(S^{\sigma}\right)=L\left(S^{\tau}\right)$ for each submodule $S$ of $A$. The proof of Lemma 3.1 (a) shows that the existence of cyclic direct summands is sufficient for $A L(S)=S$ to hold for each submodule $S$. Applying this fact we have $S^{\sigma}=S^{\tau}$.

Assume (ii) and let $\alpha=\sigma \tau^{-1}$, a one-to-one semi-linear transformation of $(F, A)$ onto itself. Then $S^{\alpha}=S$ for each submodule $S$ of $A$. If $x \neq 0$ is in $A$, from $(F x)^{\alpha}=F x$, we get $x^{\alpha}=f(x) \cdot x, f(x) \in F$. We may now follow the arguments of pp. 43-44 of [2] to conclude $f(x)=f \neq 0$, is independent of $x$ and that $x^{\alpha}=f x$ for all $x \in A$. This depends on the fact that $r(A) \geqq 2$, and in $F$ each pair of nonzero elements have a non- 
zero common left multiple. Now assume $x \neq 0$. Since $F x=(F x)^{\alpha}$, there exists $t \neq 0$ in $F$, such that $x=(t x)^{\alpha}=f(t x)=(f t) \cdot x$. Since $A$ is torsionfree $f t=1$. In a ring without proper zero divisors this implies $t f=1$ so that $f$ is a unit of $F$. But $x^{\sigma}=(f x)^{\tau}=f^{\tau} x^{\tau}=g x^{\tau}$, with $g$, a unit of $G$.

Assuming (iii) we have $x^{\alpha}=f x$ for each $x \in A$, where $\alpha=\sigma \tau^{-1}$ and $f^{\tau}=g$. Let $\eta \in E(F, A)$. Then

$$
\left(x^{\alpha}\right) \eta=(f x) \eta=f(x \eta)=(x \eta)^{\alpha},
$$

so that $\alpha \eta=\eta \alpha$ for each $\eta \in E(F, A)$. This says $\sigma \tau^{-1} \eta=\eta \sigma \tau^{-1}$ which implies $\tau^{-1} \eta \tau=\sigma^{-1} \eta \sigma$ for each $\eta \in E(F, A)$ completing the proof.

\section{REFERENCES}

1. R. Baer, Automorphism rings of primary Abelian operator groups, Ann. of Math. (2) 44 (1943), 192-227. 1952.

2. - Linear algebra and projective geometry, Academic Press, New York,

3. H. Cartan and S. Eilenberg, Homological algebra, Princeton Univ. Press, Princeton, N. J., 1956.

4. L. Fuchs, Abelian groups, Publishing House of the Hungarian Academy of Sciences, Budapest, 1958.

5. N. Jacobson, The theory of rings, Math. Surveys, No. 2, Amer. Math. Soc., Providence, R. I., 1943.

6. A. G. Kuroš, The theory of groups, Vol. 1, Chelsea, New York, 1955.

7. K. G. Wolfson, Isomorphisms of the endomorphism ring of a free module over a principal left ideal domain, Michigan Math. J. 9 (1962), 69-75.

8. - Isomorphisms of the endomorphism rings of torsion-free modules, Proc. Amer. Math. Soc. 13 (1962), 712-714.

Rutgers, The State University 